\title{
The Measurement of Everyday Cognition (ECog): Development and validation of a short form
}

\author{
Sarah Tomaszewski Farias, Ph.D. ${ }^{1}$, Dan Mungas, Ph.D. ${ }^{1}$, Danielle J. Harvey, Ph.D. ${ }^{2}$, \\ Amanda Simmons, B.A. ${ }^{1}$, Bruce R. Reed, Ph.D. ${ }^{1,3}$, and Charles DeCarli, M.D. ${ }^{1}$ \\ ${ }^{1}$ University of California, Davis Departments of Neurology \\ 2 Division of Biostatistics, School of Medicine \\ ${ }^{3}$ University of California, Davis, Veterans Affairs Northern California Health Care System
}

\begin{abstract}
Background-This study describes the development and validation of a shortened version of the Everyday Cognition scales (ECog) (1), an informant-rated questionnaire designed to detect cognitive and functional decline.

Methods-External, convergent and divergent validity, and internal consistency were examined. Data was derived from informant ratings of 907 participants who were cognitively normal, had mild cognitive impairment (MCI) or dementia.

Results-Twelve items were included in the short version (ECog-12). The ECog-12 correlated with established functional measures and neuropsychological scores, only weakly with age and education, and demonstrated high internal consistency. The ECog-12 showed excellent discrimination between the dementia and normal groups ( $\mathrm{AUC}=.95, \mathrm{CI}=.94-.97)$ and showed promise in discriminating normal older adults from those with any cognitive impairment (i.e. MCI or dementia). Discrimination between MCI and normals was poor.
\end{abstract}

Conclusions-The ECog-12 shows promise as a clinical tool for assisting clinicians in identifying individuals with dementia.

\section{Keywords}

Alzheimer's disease; mild cognitive impairment; assessment of cognition; functional assessment

\section{Introduction}

As the proportion of older adults within the population increases both in the U.S. and across the world, the prevalence of cognitive impairment and dementia is also rising. It is commonly reported that in individuals 85 and older, upwards of 30-40\% have dementia (2). Mild cognitive impairment (MCI) may be even more prevalent. Despite their prevalence,

\footnotetext{
(C) 2011 Elsevier Inc. All rights reserved.

Address Correspondence to: Sarah Tomaszewski Farias, Ph.D. University of California, Davis Department of Neurology 4860 Y Street, Suite 3700 Sacramento, CA 95817 Phone: (916) 734-6442 Fax: (916) 453-9350 sarah.farias@ ucdmc.ucdavis.edu .

Conflict of interest: Funding for this study is reported in the Acknowledgement section. None of the authors stand to benefit from this study or the assessment tool that the study describes (the instrument is provided as an Appendix to this paper).

Publisher's Disclaimer: This is a PDF file of an unedited manuscript that has been accepted for publication. As a service to our customers we are providing this early version of the manuscript. The manuscript will undergo copyediting, typesetting, and review of the resulting proof before it is published in its final citable form. Please note that during the production process errors may be discovered which could affect the content, and all legal disclaimers that apply to the journal pertain.
} 
both MCI and dementia often go unrecognized. In particular, inadequate recognition of dementia in clinical practice has been well documented and widely recognized (3-5) prompting many groups to call for regular screening of dementia in clinical settings $(6,7)$. Failure to identify dementia has important implications in terms of the establishment of appropriate treatment and supportive services.

Screening for dementia, however, can present challenges within a busy clinical setting with competing care demands. There are a number of brief performance-based cognitive screening instruments that can be useful in this regard. The MMSE is among the best well known cognitive screening measures, but it is also well recognized to have limitations in terms of its sensitivity to early disease and, like other cognitive tests, it can be confounded by low education and other demographic variables. Other performance-based cognitive screening tests have been developed that are, in some cases, briefer (i.e. Clock Drawing, 7 Minute Screen (8)) and/or have improved sensitivity to mild impairments in domains other than memory (i.e. MOCA, (9)). The use of caregiver/informant ratings of everyday cognition and daily function offer an alternative or complimentary approach to detecting neurodegenerative diseases of cognitive aging. Some of the benefits of this type of assessment are that they are time and cost efficient and can overcome some of the limitations of cognitive measures in that they are not as strongly affected by non-disease variables such as educational attainment (10-12).

The detection and characterization of functional impairments in particular, has many important clinical and research applications. It is important to identify individuals who are having difficulty functioning in their daily life because additional support or a higher level of care may be warranted. Loss of functional abilities is part of the diagnostic criteria for a dementia syndrome and is therefore important to document for diagnostic purposes. Mild changes in daily function and everyday cognition are now recognized to frequently occur early in neurodegenerative diseases of aging, including during the stage of Mild Cognitive Impairment (MCI) $(13,14)$. Importantly, the presence of problems in everyday function have been shown to help predict who will more rapidly decline and convert to dementia (10, 15). As such, informant ratings of everyday function may also have prognostic value. Finally, everyday function is also a critical outcome in tracking disease progression in clinical contexts and treatment trials (16). Measures of functional capacity are increasingly being included as endpoints in both pharmacological and nonpharmacological interventions.

A number of informant-rated measures of everyday function currently exist. However, many older instruments that are still in wide spread use were developed with little attention paid to their psychometric properties (17). Additionally, many functional instruments focus primarily on the loss of independence in basic and instrumental activities of daily living (17, 18). Presently, there are few instruments available to systematically assess more mild problems in everyday function and cognition. Those that do exist tend to be limited by restricted content coverage (19-21).

There is a clear consensus in the field that new approaches to measuring everyday function are needed $(22,23)$. The Everyday Cognition (ECog) scales were developed in response to some of the limitations of existing instruments. One goal in developing the ECog was to measure relatively mild functional changes that may predate loss of independence in major activities of daily living. A second aim in developing the ECog was to assess functional abilities that are clearly linked to specific cognitive abilities, in other words, the everyday correlates of specific neuropsychological impairments. In part this is important because some functional losses in basic and instrumental activities of daily living may be related to physical impairments rather than cognitive changes. Previous validation studies using the 
full length 39 item version of the ECog support its content, construct and external validity (1).

The present paper describes the development and validation of a shortened version of the Everyday Cognition scales (ECog). The goal of the present study was to determine if we could reduce the number of items of the original scale, while maintaining good psychometric properties. The resulting scale was then compared to the original version with respect to indices of external, convergent and divergent validity and internal consistency.

\section{Methods}

\subsection{Study participants}

Participants were drawn from a consecutive series of individuals evaluated through a university based Alzheimer's Disease Research Center (ADRC). Participants were either referred for a clinical evaluation or recruited directly from the community (see previous publication for more detail (24)). All participants received the same type of multidisciplinary diagnostic evaluation which included a detailed medical history, physical/neurological exam, lab work, neuroimaging, and neuropsychological testing. Diagnosis (Normal, MCI, Dementia) was made according to standardized criteria at a consensus case conference. Cognitive impairment was clinically identified when a participant's performance fell approximately 1.5 standard deviations below age-corrected norms and in reference to their education and occupational background. Dementia was diagnosed using DSM-III R (25) criteria for dementia. Individuals with cognitive changes not meeting criteria for a dementia were diagnosed with MCI. All diagnoses were made blind to the results of the ECog. Cases without a diagnosis were not included in the study. All procedures that were part of this study were approved by the Institutional Review Board (IRB) at the University of California, Davis and all participants consented to the study.

\subsection{Everyday Cognition (ECog)}

The original version of the ECog is an informant-based measure of cognitively-relevant everyday abilities comprised of 39 items, covering six cognitively-relevant domains: Everyday Memory, Everyday Language, Everyday Visuospatial Abilities, and Everyday Planning, Everyday Organization, and Everyday Divided Attention. For each item, informants compare the participant's current level of everyday functioning with how he or she functioned 10 years earlier. In this way, individuals serve as their own control. Ratings are made on a four-point scale: $1=$ better or no change compared to 10 years earlier, $2=$ questionable/occasionally worse, $3=$ consistently a little worse, $4=$ consistently much worse. The original ECog was developed through a rigorous process that included initial pilot testing of a larger potential pool of items. A goal of that initial pilot testing was to identify and discard items with obvious poor psychometric properties. For example, items were not retained if they were associated with a high percentage of "I don't know" responses - indicating the item did not readily apply to many individuals or was not frequently observed by an informant. However, few functional abilities will universally apply to all individuals so an 'I don't know' response option was retained. The original version has been shown to have excellent psychometric properties including good test-retest reliability $(\mathrm{r}=$. $82, \mathrm{p}<.001)$ as well as evidence of various aspects of validity including content, construction, convergent and divergent, and external validity (1). The current paper focuses on a total ECog score which is the sum of all completed items, divided by the number of items completed; thus, total scores range from 1-4. 


\subsection{Standard Measures of daily function}

The Blessed Dementia Rating Scale (BDRS) $(26,27)$ is a widely used instrument to measure functional activities and is based on a structured interview with an informant. It consists of 22 items assessing functional activities including basic activities of daily living (ADLs) (e.g., eating, dressing) and instrumental ADLs (e.g. housekeeping and money management). Ratings for each item range from $0=$ normal, $.5=$ has some trouble, and $1=$ unable to complete. The BDRS has been shown to correlate with postmortem neuropathological changes (26-29).

The Clinical Dementia Rating Scale (CDR) (30) is a structured caregiver interview measuring everyday functions related memory, orientation, judgment and problem solving, community affairs, home and hobbies, and personal care. The "sum of boxes" score was used, which is the arithmetic sum of the six subscores and represents a global measure of everyday functioning. Lower scores represent a higher level of function in daily life.

\subsection{Neuropsychological Measures}

The Spanish and English Neuropsychological Assessment Scales (SENAS) $(31,32)$ were used to measure two specific domains of cognitive functioning: episodic memory and executive function. Episodic memory is a composite variable made up of indices from a supra-span word list learning task. The executive functioning variable is comprised of several working memory and fluency tests. The SENAS subtests are psychometrically matched so that they have linear measurement properties and similar reliability of measurement.

\subsection{Scale development and analysis}

An empirical approach was used to select items for retention in a short form of the ECog. Item response theory (IRT) based analysis was used to identify which items of the original scale to include in a shortened version of the ECog. IRT is a modern approach to psychometric test development that encompasses a mathematical theory for characterizing item and scale measurement parameters and associated numerical methods for estimating these parameters. Two basic parameters of IRT models are item difficulty and discrimination. Item difficulty corresponds to the ability level at which an item is maximally discriminating. Ability is an index of examinee performance on the underlying construct that the test is intended to measure. Item discrimination refers to the degree to which small differences in ability are associated with different probabilities of passing the item. An IRT analysis simultaneously estimates difficulty and discrimination parameters for all items and ability estimates for all examinees. Item difficulty parameters and examinee ability estimates are measured on the same scale, and consequently, item difficulty corresponds to the examinee ability value at which the item is maximally discriminating. Selecting items for a scale that have high discrimination values but that have broadly variable difficulty values results in a measure that is sensitive to individual differences and change in ability across a broad range of ability, and this is important in limiting floor and ceiling effects.

Previous work with the ECog showed that the overall pool of 39 items was sufficiently unidimensional to meet fundamental IRT assumptions (1). To identify items for an abbreviated ECog, we examined estimates of item difficulty from an IRT analysis of all 39 of the original ECog items and selected the two items in each of the six ECog domains which had the highest and lowest item difficulty estimates, respectively. This was done to ensure that the shortened version of the ECog would reflect individual differences across multiple cognitive domains and would continue to be sensitive across a wide range of everyday ability, capturing both early and later occurring functional impairments. This process resulted in a new scale composed of 12 items (ECog-12). An ECog-12 score was calculated 
by averaging the scores of the 12 selected items and an ECog-39 score was similarly calculated by averaging all 39 items.

Diagnostic group differences were examined across the two versions of the ECog using oneway analysis of variance (ANOVA). A significant F statistic was followed up by pairwise comparisons using Tukey's honestly significant difference tests. Receiver operator characteristic (ROC) curves and the area under the ROC curve (AUC) were generated to examine the ability of each version of the ECog to discriminate between cognitively normal older adults and cognitively impaired individuals (i.e. those with either MCI or dementia). Analyses were also repeated to determine the discriminative properties of the scales to differentiate normals from MCI, and MCI from dementia. Follow-up analysis also examined comparisons with a mild dementia group (defined as individuals with a diagnosis of dementia who had an MMSE > 20). Sensitivity and specificity values for the ECog-12 and the original length version of the ECog (ECog-39) were generated from the above analysis (setting sensitivity at $80 \%$ to determine optimal specificity and cut-off scores). Convergent and divergent validity were examined by evaluating the strength of the correlation coefficients between the two ECog scales with other functional and cognitive measures, as well as demographics. Internal consistency was measured using Cronbach's alpha. Box plots of scores on each version of the ECog were generated to examine range of variability in scores across diagnostic groups. The appendix contains the items that make up the 12-item version.

\section{Results}

\subsection{Sample Characteristics}

Results were derived from informant ratings for 907 elderly participants. The sample was comprised of a cognitively diverse group that included 193 participants who were cognitively normal 190 who had MCI, 458 who had been diagnosed with a dementia; an additional 66 individuals had a diagnosis of Questionable Cognitive Impairment (this group was not included in analyses comparing diagnostic groups because they did not clearly fit into the normal or MCI groups). Of those with dementia, the majority had possible or probable Alzheimer's disease. Years of education ranged from 0 to over 20 yeas of education (mean for the entire sample $=13.84$ (3.71)) with 124 individuals having less than 12 years of education. Participants were of various racial/ethnic backgrounds with about a quarter of the sample being non-Caucasian: 134 (15\%) were Hispanic, 95 (10\%) were African American, 616 (68\%) were Caucasian, and the remainder of the sample was of another or unknown ethnicity. Table 1 presents the characteristics of the sample by diagnostic category.

In terms of informant characteristics, $48 \%$ of the informants were a spouse, $39 \%$ were an adult child of the participant, or a son-in-law/daughter-in-law, 5\% were another family member, $5 \%$ were friends, and the remainder were reported as 'other.' Sixty-three percent saw the participant on a daily basis, $92 \%$ on at least a weekly basis. Average hours per week spent with the participant was $87.3(\mathrm{SD}=70.1)$.

\subsection{Scale differences across diagnostic groups (external validity)}

The average total score on the ECog-12 score was significantly different across the three diagnostic syndromes ( $\mathrm{p}<.001)$; follow-up comparisons adjusted for multiple comparisons further showed that each group was significantly different from the other two groups. The ECog-39 total score also differed for each diagnostic group (p's <.05, adjusted for multiple comparisons). Table 1 provides the mean ECog total score for both versions across diagnostic syndromes. Analyses below also examined mild dementia separately (defined as 
and MMSE score greater than 20); the mean total score for the mild dementia group on the ECog-12 was 3.21 (.69) and for the ECog-39 it was $2.80(.70)$.

ROC curves were generated to measure the effectiveness of each ECog version to classify patients into diagnostic groups. The ECog-12 was equally as sensitive as the full length version in discriminating between those with any cognitive impairment (either dementia or $\mathrm{MCI}$ ) and participants with normal cognitive function. The area under the curve (AUC) for both versions of the ECog was .91 (95\% CI = .88 -.93) (see also Table 2). Optimal cut off scores for a given sensitivity and specificity are also provided in Table 2. Figure 1 plots the ROC curve for the discrimination between individuals with cognitive impairment versus cognitively normal elderly.

We also examined the ability of both versions of the ECog to discriminate between other various diagnostic groupings (see Table 2). Not surprisingly, the discrimination between individuals with dementia versus the cognitively normal group was highest with an AUC of . 95 for the ECog-12. The full length and short form of the ECog showed almost identical results in this comparison. Discrimination of the dementia group from individuals with MCI was less strong (AUC $=.80$ for the ECog-12) with a specificity value somewhat higher for the full length version of the ECog. The ability of both version of the ECog to discriminate those with MCI from normal elderly was weakest (AUC $=.79$ for the ECog-12) with relatively low specificity values.

Moderate to severe dementia is relatively easier for clinicians to identify while identifying those with mild dementia or MCI from normal elders remains challenging. As such, we reran several of the above comparisons including only those with mild dementia. The discrimination of mild dementia from normal elderly was still quite good and nearly identical as compared to the results of the analyses that included the more severely demented individuals ( $\mathrm{AUC}=.94$ for the ECog-12). However, the discrimination between mild dementia and MCI was poor, particularly with the short version of the ECog.

\subsection{Relationship with other variables (convergent and divergent validity)}

Convergent validity was assessed by examining the relationship between the ECog-12 and other standard measures of daily function (see Table 3 which provides $\mathrm{R}^{2}$ values). The ECog-12 showed a moderate to strong correlation with existing measures of daily function, including the CDR (sum of boxes) and the Blessed Roth Dementia Rating Scale. While everyday function is expected to be related to measures of cognitive function, the relationship between the ECog-12 and the neuropsychological variables was anticipated to be weaker than the relationship between the ECog-12 and other measures of everyday function. As expected in this regard, the ECog-12 showed moderate correlations with neuropsychological tests of episodic memory and executive function. The relationships between the original version of the ECog and both of the existing functional scales and the neuropsychological measures were of a similar magnitude although somewhat higher when compared to the relationship between these same variables and the ECog-12.

Finally, the association between the ECog-12 and select demographic variables was also examined (divergent validity). Here it was anticipated that there would be negligible associations. Results show that not more than $4 \%$ of the variance in the ECog-12 can be accounted for by either age or education level. Again, the magnitude of the relationship between the ECog-12 and the ECog-39 and these demographic variables was similar.

\subsection{Internal consistency and other psychometric characteristics}

Cronbach's alpha was calculated as a measure of internal consistency. Cronbach's alpha for the ECog-12 was .96, supporting its use as a global measure of everyday cognition. Figure 2 
presents box plots of the ECog-12 in comparison to the total score for the ECog-39, showing the median score, and upper and lower quartiles for each diagnostic group. First, the figure demonstrates that both versions of the ECog do not show appreciable ceiling effects for the MCI group, and even to a large extent in the normal group. This is in contrast to some existing functional measures on which individuals with early disease show little to no loss in the functional abilities measured. However, there is some overlap in the distribution of scores between the normal and MCI groups on both version of the ECog, making crosssectional discrimination of these two groups difficult using the ECog alone. Additionally, in contrast to the full length version, the ECog-12 does show somewhat of a floor effect in the dementia group, wherein $26 \%$ of the dementia sample is scoring at the maximally impaired level. This is compared to $5 \%$ of the dementia sample who score at the maximum level on the original version of the ECog. Those individuals who scored at the maximal level on the ECog-12 had a mean MMSE of 14.5, and a CDR sum of boxes mean of 9.72, suggesting they were showing a fairly high level of cognitive and functional impairment.

Finally, we examined the percent of items on which the informant indicated that they could not rate the particular functional ability (i.e. they circled the "I don't know" response option). For the 12 items included in the short form of the ECog, the percentage of 'I don't know' responses ranged from $0 \%$ to $8 \%$ on 10 of the 12 items; on the items assessing the ability to read a map and to balance a checkbook, about $20 \%$ of informants indicated that they could not rate those items (not the same informants in each case although there was some overlap). Informants who spent less time with the participant were less likely to rate the map reading item but this was not the case for the checkbook item.

\section{Discussion}

The strongest evidence of the utility of the ECog-12 to separate groups comes from the comparison between the cognitively normal group and individuals with dementia. Here the AUC was high (.95) and sensitivity/specificity was excellent. Excluding cases of moderate to severe dementia affect the accuracy of this discrimination very little, suggesting that individuals at the extreme end of the impairment spectrum were not artificially inflating this discrimination. Recent reports indicate that dementia often goes unrecognized in clinical setting, and that one obstacle to identifying dementia is the difficulty clinicians have in discriminating it from cognitive problems attributed to normal aging (33). To this end the ECog-12 provides clinicians with another tool to help differentiate individuals with dementia from normal older adults.

In clinical settings it is also often important to identify individuals at increased risk for developing dementia in the near future. The presence of MCI has been widely shown to increase risk of future dementia. Results suggest that the ECog may also have some utility in helping to discriminate individuals with either MCI or dementia from healthy older adults; using the more conservative comparison between mild dementia/MCI versus normal controls, sensitivity and specificity values were $80 \%$ and $78 \%$, respectively $(\mathrm{AUC}=.88$ ). The ability of the ECog-12 to make finer discriminations is not well supported in this study. Specifically, the relative specificity values for distinguishing either MCI from normal elderly or from mild dementia were relatively low. While it would be particularly useful to develop brief screening tools that would accurately discriminate individuals with MCI from healthy elderly, this has proven difficult using a variety of other screening tools. Both performance-based cognitive screening tools $(34,35)$ and informant-rated instruments have yielded relatively poor discrimination between MCI and healthy elders (36). The combination of both informant rating scales and brief performance-based testing is likely to yield better discrimination between these groups than either one alone (37). Utilizing data from both the ECog-12 and the MMSE did improve the discrimination of individuals with 
MCI from healthy elders (at $80 \%$ sensitivity, specificity was $76 \%$ when combining the two types of information (data not shown) as compared to $62 \%$ specificity with the ECog-12 alone). Use of a more sensitive performance-based instrument, in combination with the ECog-12, will likely yield even better results.

The ECog-12 offers a number of advantages over other available screening tools. In contrast to performance-based cognitive screening tools, the current results show that the ECog-12 is affected minimally by educational background. This may, in part, be due to the fact that individuals are rated in comparison to their own baseline. Further, many performance-based cognitive tests are affected by cultural differences among groups, which can further confound the test results, making them difficult to interpret. In previous work, the ECog was shown to be affected very little by ethnicity (1). As such, the ECog-12 may be helpful in screening for dementia in low education or minority patients, although further validation studies with other racial/ethnic groups are needed.

Neither the ECog-12 nor the original version show the ceiling effects that many other functional and cognitive screening instruments show in very mildly impaired older adults, which may facilitate its use in tracking functional change over time. However, the dementia group did show a restriction in range of scores on the ECog-12 as compared to the original length version, such that about a quarter of the participants diagnosed with a dementia syndrome were at the maximal level of impairment as measured by the ECog-12. This is in contrast to only about $5 \%$ of the dementia sample scoring at floor level on the original version of the ECog. As a result, whether a clinician or researcher chooses to use the abbreviated versus original length version of the ECog will depend on the goals of the assessment, and the characteristics of the sample under study. If longitudinal tracking over a wide range of ability level and disease severity is desired, the original length version would be preferable.

The methods used to select items for this short form of the ECog are different than those typically employed. All items selected had high discrimination values. Higher discrimination is associated with a lower standard error of measurement, or equivalently, higher reliability. Additionally, items were also selected based on their associated difficulty parameters such that items with either a high difficulty value or a relatively low difficulty value were systematically selected, the goal being to select items that would cover a fairly broad range of ability level.

The original ECog was designed to measure specific aspects of everyday cognition related to memory, language, visuospatial functions, and executive functioning and previous confirmatory factor analysis using the full length version of the ECog supported this multidimensional structure (1). However, the ECog-12 is likely to be best used as a global measure of everyday function since the total number of items assessing each specific domain has been substantially reduced. Thus, the trade off for the increased brevity is decreased breadth and depth of assessment of the specific domains of everyday cognition. The current results showing good internal consistency of the ECog-12 support its use as a global measure of everyday cognition.

There are a number of limitations to the study which deserve mention. The sample is weighted in favor of cognitively impaired persons and the distribution of individuals with normal cognition, MCI and dementia in the current sample differ from base rates of these syndromes within the elderly population at large. This may inflate the performance of tests for impairment and calls for population-based applications for further validation.

Additionally, many although not all of the individuals in this sample were enrolled in longitudinal research; such individuals tend to have a lower burden of medical illness than 
many older adults in the community, this also presents a limit to the generalizability of findings. About a quarter of the sample was of an ethnic/racial minority. Additionally, although the mean education level of the sample was relatively high (about 13.8 years), nearly a quarter of the sample had less than a high school education. Still, results may not generalize to individuals who differ substantially from the sample in this study.

There are also limitations to relying on the reports of informants; such ratings can be subject to the effects of systematic bias. For example, informant characteristics such as mood or degree of caregiver burden can affect ratings $(21,38)$. Informant report has, however, been shown to reliably differentiate individuals with and without dementia, and such information can be useful in predicting who will go on to further decline $(15,39)$. Not all patients seen in primary care, or other clinical settings attend appointments with an informant and so such ratings may be difficult or impossible to obtain (40). Additionally, some functional abilities may be relatively more difficult for informants to rate (i.e. navigational skills or financial abilities). Self-reported everyday function may offer another alternative to informant report, although self report has been widely reported as unreliable in individuals with frank dementia, in large part due to diminished awareness of deficits. The use of self report to identify more mild degrees of cognitive and functional loss has yielded inconsistent results $(36,37)$, but may be helpful in very early disease (41).

It is widely recognized that dementia often goes undetected in primary care settings. While there are a wide variety of performance-based cognitive screening tools (see (42) for a review) there are comparatively few instruments that assess everyday functional abilities. The ECog-12 is a very brief informant-rated questionnaire that assesses cognitively-relevant functional abilities. In most respects it shows similar psychometric properties to the original 39 -item version. The present results suggest that the ECog-12 provides clinicians with another tool to help them differentiate older adults with dementia from those who are cognitively normal. The ECog- 12 can be completed by an informant within just a few minutes either prior to a clinic office visit with the treatment provider (i.e. in the waiting room or mailed in ahead of time) or while the clinician is completing a physical exam or obtaining other information directly from the patient. To facilitate clinical usefulness we have provided suggested cut-off scores that can be used to help a clinician interpret the results of the ECog-12. However, it is important to keep in mind that cut off points are dependent on the degree to which sensitivity versus specificity is valued. Further crossvalidation in an independent sample will be needed to verify the utility of these cut off values. Additionally, the usefulness of the ECog-12 for longitudinally tracking functional decline, and in identifying those on a worse trajectory should be the focus of future research.

\section{Acknowledgments}

This study was supported by the following grants from the NIA: AG031252, AG010220, AG031563, AG10129. All authors had full access to the data and take responsibility for the integrity of the data and it's analysis.

\section{Appendix}

Items included in the ECog-12

Remembering where he/she has placed objects.

Remembering the current date or day of the week.

Communicating thoughts in a conversation.

Understanding spoken directions or instructions. 
Reading a map and helping with directions when someone else is driving.

Finding one's way around a house/building that he/she has visited many times.

Anticipating weather changes and planning accordingly.

Thinking ahead.

Keeping living and work space organized.

Balancing the checkbook/account without error.

Doing two things at once.

Cooking or working, and talking at the same time.

\section{References}

1. Farias, S Tomaszewski; Mungas, D.; Reed, B.; Cahn-Weiner, D.; Jagust, W.; Baynes, K., et al. The measurement of everyday cognition (ECog): Scale development and psychometric properties. Neuropsychology. 2008; 22:531-544. [PubMed: 18590364]

2. Evans DA, Scherr PA, Cook NR. Estimated prevalence of Alzheimer'S disease in the United States. Milbank Q. 1990; 68:267-289. [PubMed: 2233632]

3. Sterberg SA, Wolfson C, Baumgarten M. Undetected dementia in community-dwelling older people: the Canadian Study of Health and Agng. J Am Geriatr Soc. 2000; 48:1430-1434. [PubMed: 11083319]

4. Valcour VG, Masaki KH, Curb JD, Blanchette PL. The detection of dementia in primary care setting. Arch Intern Med. 2000; 160:2964-2968. [PubMed: 11041904]

5. Callahan CM, Hendrie HC, Tierney WM. Documentation and evaluation of cognitive impairment in elderly primary care patients. Ann Intern Med. 1995; 122:422-429. [PubMed: 7856990]

6. Ashford JW, Borson S, O'Hara R, Dash P, Frank L, Robert P, et al. Should older adults be screened for dementia? It is important to screen for evidence of dementia! Alzheimer's \& Dementia. 2007; 3:75-80.

7. Solomon PR, Murphy CA. Should we screen for Alzheimer's disease? A review of the evidence for and against screening for Alzheimer's disease in primary care practice. Geriatrics. 2005; 60:26-31. [PubMed: 16287338]

8. Meulen EFJ, Schmand B, van Campen JP, de Koning SJ, Ponds RW, Scheltens P, et al. The seven minute screen: a neurocognitive screening test highly sensitive to various types of dementia. $J$ Neurol Neurosurg Psychiatry. 2004; 75:700-705. [PubMed: 15090563]

9. Nasreddine ZS, Phillips NA, Charbonneau S, Whitehead V, Collin I, Cummings JL, et al. The Montreal Cognitive Assessment (MoCA): a brief screening tool for Mild Cognitive Impairment. J Am Geriatri Soc. 2005; 53:695-699.

10. Farias, S Tomaszewski; Cahn-Weiner, D.; Harvey, D.; Reed, B.; Mungas, D.; Kramer, JH., et al. Longitudinal changes in memory and executive functioning are associated with longitudinal change in instrumental activities of daily living in older adults. Clin Neuropsychol. 2009; 23:446461. [PubMed: 18821181]

11. Jorm AF, Jacomb PA. The Informant Questionnaire on Cognitive Decline in the Elderly (IQCODE): Socio-demographic correlates, reliability, validity and some norms. Psychological Medicine. 1989; 19:1015-1022. [PubMed: 2594878]

12. Del-Ser T, Morales JM, Barguero MS, Canton R, Bermejo F. Application of the Spanish version of the "Informant Questionnaire on Cognitive Decline in the Elderly" in the clinical assessment of dementia. Alzheimer Dis Assoc Disord. 1997; 11:3-8. [PubMed: 9071438]

13. Farias, S Tomaszewski; Mungas, D.; Reed, B.; Harvey, D.; Cahn-Weiner, D.; DeCarli, C. MCI is associated with deficits in everyday functioning. Alzheimer Dis Assoc Disord. 2006; 20:217-223. [PubMed: 17132965] 
14. Perneczky R, Pohl C, Sorg C, Hartmann J, Tosic N, Grimmer T, et al. Impairment of activities of daily living requiring memory or complex reasoning as part of the MCI syndrome. Int J Geriatr Psychiatry. 2006; 21:158-162. [PubMed: 16416470]

15. Daly E, Zaitchik D, Copeland M, Schmahmann J, Gunther J, Albert M. Predicting conversion to Alzheimer disease using standardized clinical information. Arch Neurol. 2000; 57:675-680. [PubMed: 10815133]

16. Rockwood K. The measuring, meaning, and importance of activities of daily living (ADLs) as an outcome. International Psychogeriatrics. 2007; 19:467-482. [PubMed: 17359560]

17. Lawton MP, Brody EM. Assessment of older people: Self-maintaining and instrumental activities of daily living. The Gerontologist. 1969; 9:179-186. [PubMed: 5349366]

18. Galasko D, Bennett DA, Sano M, Ernesto C, Thomas R, Grundman M, et al. An inventory to assess activities of daily living for clinical trials in Alzheimer's Disease. The Alzheimer's Disease Cooperative Study. Alzheimer Dis Assoc Disord. 1997; 11(Suppl 2):S33-39.

19. Galvin J, Roe C, Xiong C, Morris J. Validity and reliability of the AD8 informant interview in dementia. Neurology. 2006; 67:1942-1948. [PubMed: 17159098]

20. Jorm AF, Scott R, Cullen JS, MacKinnon AJ. Performance of the Informant Questionnaire on Cognitive Decline in the Olderly (IQCODE) as a screening test for dementia. Psychological Medicine. 1991; 21:785-790. [PubMed: 1946866]

21. Jorm AF. A short form of the Informant Questionnaire on Cognitive Decline in the Elderly (IQCODE): Development and cross-validation. Psychological Medicine. 1994; 24:145-153. [PubMed: 8208879]

22. Broich K. Outcome measures in clinical trials on medicinal products for the treatment of dementia: A European regulatory perspective. Int Psychogeriatr. 2007; 19:509-524. [PubMed: 17433121]

23. Massoud F. The role of functional assessment as an outcome measure in antidementia treatment. Can J Neurol Sci. 2007; 34:S47-S51. [PubMed: 17469682]

24. Farias, S Tomaszewski; Mungas, D.; Reed, B.; Harvey, D.; DeCarli, C. Progression of mild cognitive impairment to dementia in clinic- vs. community-based cohorts. Archives of Neurology. 2009; 66:1151-1157. [PubMed: 19752306]

25. American Psychiatric Association. Diagnostic and statistical manual of mental disorders. Rev. 3rd ed. American Psychiatric Association; Washington DC: 1987.

26. Blessed GT, Tomlinson BE, Roth M. The association between quantitative measures of dementia and of senile change in the cerebral grey matter of elderly subjects. Br J Psychiatry. 1968; 114:797-811. [PubMed: 5662937]

27. Blessed GT, Tomlinson BE, Roth M. Blessed-Roth Dementia Scale. Psychopharm Bul. 1988; 24:705-708.

28. Stern Y, Mayeux R, Sano M. Predictors of disease course in patients with probable Alzheimer's disease. Neurology. 1987; 37:1649-1653. [PubMed: 3658173]

29. Stern Y, Hesdorffer D, Sano M. Measurement and prediction of functional capacity in Alzheimer's disease. Neurology. 1990; 40:8-14. [PubMed: 2296387]

30. Morris JC. The Clinical Dementia Rating (CDR): Current version and scoring rules. Neurology. $1993 ; 43: 24 ` 21-2414$.

31. Mungas D, Reed B, Crane P, Haan M, Gonzales H. Spanish and English Neuropsychological Assessment Scales (SENAS): Further development and psychometric characteristics. Psychological Assessment. 2004; 16:347-359. [PubMed: 15584794]

32. Mungas D, Reed B, Marshall S, Gonzales H. Development of psychometrically matched English and Spanish neuropsychological tests for older persons. Neuropsychol. 2000; 14:209-223.

33. Knopman D, Donohue JA, Gutterman EM. Patterns of care in the early stages of Alzheimer's disease: impediments to timely diagnosis. J Am Geriatr Soc. 2000; 48:300-304. [PubMed: 10733057]

34. Lavery LL, Lu SY, Chang CC, Saxton J, Ganguli M. Cognitive assessment of older primary care patients with and without memory complaints. J Gen Intern Med. 2007; 22:949-954. [PubMed: 17453265] 
35. Forti P, Olivelli V, Rietti E, Maltoni B, Ravaglia G. Diagnostic performance of an Executive Clock Drawing Task (CLOX) as a screening tool for mild cognitive impairment in elderly persons with cognitive complaints. Dement Geriatr Cogn Disord. 2010; 30:20-27. [PubMed: 20606441]

36. Schinka JA, Brown LM, Proctor-Weber Z. Measuring change in everyday cognition: Development and initial validation of the Cognitive Change Checklist (3CL). Am J Geriatr Psychiatry. 2009; 17:516-525. [PubMed: 19461260]

37. Galvin JE, Roe C, Morris JC. Evaluation of cognitive impairment in older adults: Combining brief informant and performance measures. Arch Neurol. 2007; 64:718-724. [PubMed: 17502471]

38. Teri L. Behavior and caregiver burden: Behavioral problems in patients with Alzheimer's disease and its association with caregiver distress. Alzheimer Dis Assoc Disord. 1997; 11:S35-S38. [PubMed: 9339271]

39. Monnot M, Brosey M, Ross E. Screening for dementia: Family caregiver questionnaires reliably predict dementia. J Am Board Fam Prac. 2005; 18:240-256.

40. Wolff JL, Roter DL. Hidden in plain sight: medical visit companions as resources for vulnerable older adults. Arch Intern Med. 2008; 168:1409-1415. [PubMed: 18625921]

41. Saykin AJ, Wishart HA, Rabin LA, Santulli RB, Flashman LA, Waest JD, et al. Older adults with cognitive complaints show brain atrophy similar to that of amnestic MCI. Neurology. 2006; 67:834-842. [PubMed: 16966547]

42. Holsinger T, Deveau J, Boustani M, Williams JW. Does this patient have dementia? JAMA. 2007; 297:2391-s2404. [PubMed: 17551132] 


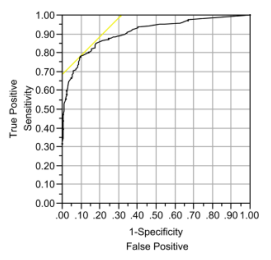

Figure 1. Receiver operating characteristic curve for the ECog-12

Receiver operating characteristic curve for the ECog-12 discriminating individuals with cognitive impairment (either MCI or dementia) from cognitively normal elderly. 


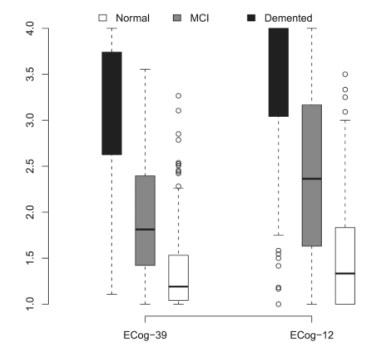

Figure 2. Range of variability of ECog scores

Box plots of the total scores for ECog-39 (original version) and the ECog-12, showing the median, and upper and lower quartiles for each clinical group. 
Table 1

Demographic and mean (standard deviation) cognitive and functional scores by diagnostic group.

\begin{tabular}{|l|l|l|l|}
\hline & $\begin{array}{l}\text { Normals } \\
(\mathbf{N}=\mathbf{2 5 9})\end{array}$ & $\begin{array}{l}\text { MCI } \\
(\mathbf{N = ~ 1 9 0 )}\end{array}$ & $\begin{array}{l}\text { Dementia } \\
(\mathbf{N = ~ 4 5 8})\end{array}$ \\
\hline Age & $74.2(8.0)$ & $76.7(7.7)$ & $77.5(8.1)$ \\
\hline Gender (\% female) & $62 \%$ & $50 \%$ & $60 \%$ \\
\hline Education (years) & $14.6(3.2)$ & $14.2(4.0)$ & $13.4(3.7)$ \\
\hline MMSE & $29.04(7.90)$ & $26.48(6.18)$ & $20.94(15.48)$ \\
\hline CDR & $.41(.80$ & $1.87(1.49)$ & $6.71(4.22)$ \\
\hline${ }^{*}$ Episodic Memory & $.14(.76)$ & $-.82(.67)$ & $-.1 .57(.63)$ \\
\hline${ }^{*}$ Executive Function & $.10(.61)$ & $-.31(.56)$ & $-.97(.70)$ \\
\hline ECog-12 & $1.55(.61)$ & $2.44(.92)$ & $3.41(.72)$ \\
\hline ECog-39 & $1.37(.46)$ & $1.94(.66)$ & $3.06(.76)$ \\
\hline
\end{tabular}

Values reported are $\mathrm{Z}$ scores (mean $=0.0$, standard deviation $=1.0$ ); the 66 participants with Questionable Cognitive Impairment are not included in this table. 
Table 2

Discrimination between diagnostic groups for each version of the ECog.

\begin{tabular}{|c|c|c|c|c|}
\hline & Test & AUC & $\begin{array}{l}\text { Specificity } \\
\text { (with 80\% } \\
\text { sensitivity) }\end{array}$ & $\begin{array}{l}\text { Optimal cut-off } \\
\text { score }^{*}\end{array}$ \\
\hline \multirow{2}{*}{$\begin{array}{l}\text { Impaired*vs. } \\
\text { Normal }\end{array}$} & ECog-39 & $.91(\mathrm{CI}=.88-.93)$ & $84 \%$ & 1.81 \\
\hline & ECog-12 & $.91(\mathrm{CI}=.88-.93)$ & $81 \%$ & 2.30 \\
\hline \multirow{2}{*}{$\begin{array}{l}\text { Dementia vs. } \\
\text { Normal }\end{array}$} & ECog-39 & $.96(\mathrm{CI}=.94-.97)$ & $95 \%$ & 2.34 \\
\hline & ECog-12 & $.95(\mathrm{CI}=.94-.97)$ & $95 \%$ & 2.83 \\
\hline \multirow{2}{*}{$\begin{array}{l}\text { Dementia vs. } \\
\text { MCI }\end{array}$} & ECog-39 & $.85(\mathrm{CI}=.82-.89)$ & $74 \%$ & 2.35 \\
\hline & ECog-12 & $.80(\mathrm{CI}=.76-.84)$ & $64 \%$ & 2.83 \\
\hline \multirow{2}{*}{$\begin{array}{l}\text { MCI vs. } \\
\text { Normal }\end{array}$} & ECog-39 & $.79(\mathrm{CI}=.74-.83)$ & $61 \%$ & 1.32 \\
\hline & ECog-12 & $.79(\mathrm{CI}=.74-.84)$ & $62 \%$ & 1.54 \\
\hline \multirow{2}{*}{$\begin{array}{l}\text { Impaired } 2^{* *} \\
\text { vs. Normal }\end{array}$} & ECog-39 & $.87(\mathrm{CI}=.84-.90)$ & $77 \%$ & 1.58 \\
\hline & ECog-12 & $.88(\mathrm{CI}=.85-.91)$ & $78 \%$ & 2.0 \\
\hline \multirow{2}{*}{$\begin{array}{l}\text { Mild Dementia } \\
\text { vs. MCI }\end{array}$} & ECog-39 & $.80(\mathrm{CI}=.76-.85)$ & $74 \%$ & 2.12 \\
\hline & ECog-12 & $.74(\mathrm{CI}=.69-.79)$ & $58 \%$ & 2.70 \\
\hline \multirow{2}{*}{$\begin{array}{l}\text { Mild Dementia } \\
\text { vs. Normal }\end{array}$} & ECog-39 & $.94(\mathrm{CI}=.92-.97)$ & $91 \%$ & 2.12 \\
\hline & ECog-12 & $.94(\mathrm{CI}=.92-.97)$ & $92 \%$ & 2.70 \\
\hline
\end{tabular}

* This is based on a sum of the item scores divided by the total number of items completed by the informant (range: $1=$ no change/better performance than in the past to $4=$ consistently much worse than in the past).

Impaired $=$ persons who had a clinical diagnosis of dementia or MCI 


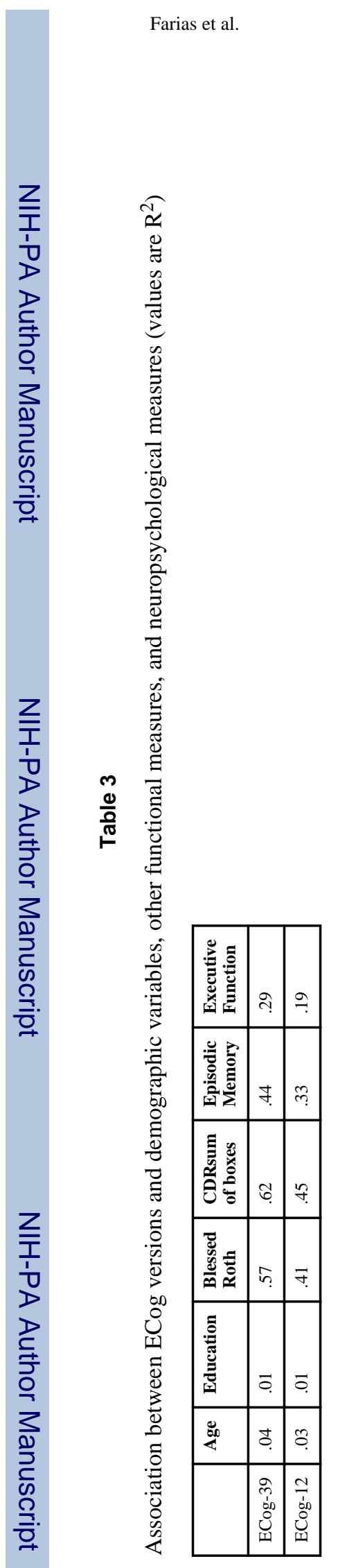

Alzheimers Dement. Author manuscript; available in PMC 2012 November 1. 